Article

\title{
Temperature-Dependent Conformation Behavior of Isolated Poly(3-hexylthiopene) Chains
}

\author{
Sanwardhini Pantawane (D) and Stephan Gekle *(D)
}

Biofluid Simulation and Modeling, Theoretische Physik VI, Universität Bayreuth, 95440 Bayreuth, Germany; Sanwardhini.Pantawane@uni-bayreuth.de

* Correspondence: stephan.gekle@uni-bayreuth.de

Citation: Pantawane, S.; Gekle, S. Temperature-Dependent Conformation Behavior of Isolated Poly(3-hexylthiopene) Chains. Polymers 2022, 14, 550. https:// doi.org/10.3390/polym14030550

Academic Editor: Luca Valentini

Received: 3 December 2021

Accepted: 25 January 2022

Published: 28 January 2022

Publisher's Note: MDPI stays neutral with regard to jurisdictional claims in published maps and institutional affiliations.

Copyright: (C) 2022 by the authors. Licensee MDPI, Basel, Switzerland. This article is an open access article distributed under the terms and conditions of the Creative Commons Attribution (CC BY) license (https:// creativecommons.org/licenses/by/ $4.0 /)$.

\begin{abstract}
We use atomistic as well as coarse-grained molecular dynamics simulations to study the conformation of a single poly(3-hexylthiopene) chain as a function of temperature. We find that mainly bundle and toroid structures appear with bundles becoming more abundant for decreasing temperatures. We compare an atomistic and a Martini-based coarse-grained model which we find in very good agreement. We further illustrate how the temperature dependence of P3HT can be connected to that of simple Lennard-Jones model polymers in a vacuum. Upon adding solvent (THF) we observe the occurrence of a prominent swelling of the molecular size at a temperature of about $220 \mathrm{~K}$. This swelling is in close agreement with the interpretation of recent spectroscopic experiments which allows us to explain the experimental observations by an increased frequency of bundle structures.
\end{abstract}

Keywords: poly(3-hexylthiopene); stiff; flexible; semi-flexible polymers; Lennard-Jones model polymers; molecular dynamics simulation

\section{Introduction}

Poly(3hexylthiophene) (P3HT) is a conjugated polymer widely used in optoelectronic devices like thin film-field effect transistors and organic photovoltaics because of its high field-effect mobilities and significant mechanical strength [1]. These favorable properties are intimately related to its molecular arrangement [2-5]. The basis for understanding this molecular arrangement is a solid understanding of the behavior of a single P3HT chain. In this respect, Heffner et al. [6] carried out dynamic light scattering experiments on isolated chains concluding that $\mathrm{P} 3 \mathrm{HT}$ behaves as a flexible polymer. More recently, neutron scattering (SANS) experiments by the authors of [7] and viscosity measurements by the authors of [8] determined the persistence length from which they suggested to classify P3HT as a semiflexible polymer instead. Besides these, refs. $[9,10]$ conducted single molecule spectroscopy experiments finding different chain conformations depending on the solvent quality. Raithel et al. [11] investigated local planarization effects and torsional order on the scale of a single emitting site. On the numerical side, in addition to their experimental work, ref. [9] conducted coarse-grained molecular dynamics (MD) simulations finding a highly anisotropic, ordered structure for regioregular $\mathrm{P} 3 \mathrm{HT}$ at temperatures below the collapse transition. Schwarz et al. [12] found similar structures and investigated their role as precursors for multi-chain aggregates while Tapping et al. [13] used coarse-grained molecular dynamics simulations in combination with exciton modeling to predict absorption and emission spectra.

A recent series of spectroscopic experiments on highly monodisperse, regioregular P3HT by Panzer et al. $[14,15]$ specifically focused on the detailed temperature dependence of the molecular arrangement. As the temperature was increased from $170 \mathrm{~K}$ to $300 \mathrm{~K}$, ref. [14] noted a shift in the absorption spectra from blue to red. These results led to the interpretation that the collapse transition from a random coil at high temperatures into a more 
regular conformation at low temperatures proceeds via an intermediate more extended structure termed a "planarized swollen coil". This interpretation was rationalized by the classical work of Kolinski et al. [16] who conducted grid-based Monte-Carlo simulations on semi-flexible model polymers and predicted a swelling of the polymer before the final collapse at low temperatures within a narrow parameter range. Interestingly, similar effects were also observed during film formation in P3HT by the same authors.

Motivated by these experiments, we here revisit the temperature-dependent singlechain behavior of P3HT using both atomistic as well as coarse-grained MD simulations. Our specific aim is to investigate explicitly the "planarized swollen coil" structure whose existence has been derived from an interpretation of the spectroscopic signatures in [14]. For this, we start off by MD simulations on the temperature-dependent collapse transition of a simple Lennard-Jones model system where we reproduce the conformations obtained in earlier works using Monte-Carlo systems [16-19]. In order to become more realistic, we then compare these model systems to MD simulations of P3HT using an atomistic force field based on the work in [20] in a vacuum. In the next step, we use these highly detailed simulations to benchmark a coarse-grained model based on the Martini force field [21], finding good agreement with the fully atomistic model. Finally, the coarse-grained model is computationally efficient enough to allow us to study the P3HT behavior in explicit THF solvent. Here, we indeed observe a molecular swelling at temperatures around $220 \mathrm{~K}$ which can be closely connected to the "planarized swollen coil" observed in the experiments by Panzer et al.

\section{Simulation Models and Details}

\subsection{Molecular Dynamics Simulations}

We use the MD simulation package GROMACS [22] (version 2018.1) for all simulations. The LINCS algorithm is used for constraining the bonds and a cutoff radius of $1.0 \mathrm{~nm}$ is used for the non-bonded potential. To maintain a constant temperature, we employ a velocity rescaling thermostat [23]. Periodic boundary conditions are applied in all three directions. One of the key quantities which we analyze in our work is the mean-squared radius of the gyration of a polymer averaged over time which is defined as

$$
\left\langle S^{2}\right\rangle=\frac{1}{n}\left\langle\sum_{i=1}^{n}\left(r_{i}-r_{C M}\right)^{2}\right\rangle
$$

where $r_{i}$ is the position of monomer $i, r_{C M}$ is the center of mass of the polymer, and \langle\rangle denotes an ensemble average over the trajectory of the MD run. Secondly, we calculate the maximum distance between the two monomers of the polymer and denote it with $D_{\max }$. The errors bars denote the standard deviation of the sampling data.

\subsection{Lennard-Jones Model Polymer}

In the Lennard-Jones (LJ) polymer each monomer is modeled as a single LJ bead of the form

$$
U_{L J}=4 \epsilon\left[\left(\frac{\sigma}{r}\right)^{12}-\left(\frac{\sigma}{r}\right)^{6}\right]=\frac{C_{12}}{r^{12}}-\frac{C_{6}}{r^{6}}
$$

$C_{12}$ was fixed at $10^{-6} \frac{\mathrm{kJ}}{\mathrm{mol} \mathrm{nm}^{12}}$ while the $C_{6}$ values were varied in order to tune the polymer stiffness as described below. Neighboring monomers are connected to each other with a harmonic potential $U_{\text {bond }}$ of the form

$$
U_{b o n d}=\frac{1}{2} k^{b}(r-b)^{2}
$$

The equilibrium distance $b$ between two monomers was set to $0.1 \mathrm{~nm}$. Angle potentials had the form

$$
U_{\text {angle }}=\frac{1}{2} k^{\theta}\left(\theta-\theta_{0}\right)^{2}
$$


with the equilibrium angle $\theta_{0}=180^{\circ}$ and the bending stiffness used as a tuning parameter.

The stiffness of the polymer was tuned in two different ways. In the first method, we fix $k^{\theta}=95.0 \frac{\mathrm{kJ}}{\mathrm{mol} \mathrm{rad}^{2}}$ and vary the attractive non bonded parameter $C_{6}$ with the stiffest polymers having the most repulsive potential $\left(C_{6}=0.0000 \frac{\mathrm{kJ}}{\mathrm{mol} \mathrm{nm}^{6}}\right)$ and the most flexible polymers having the most attractive potential $\left(C_{6}=0.001 \frac{\mathrm{kJ}}{\mathrm{mol} \mathrm{nm}^{6}}\right)$. In the second method, we fix $C_{6}=0.00045 \frac{\mathrm{kJ}}{\mathrm{mol} \mathrm{nm}^{6}}$ and vary the bending potential $k^{\theta}$ from $5.0 \frac{\mathrm{kJ}}{\mathrm{mol} \mathrm{rad}^{2}}$ to $250.0 \frac{\mathrm{kJ}}{\mathrm{mol} \mathrm{rad}^{2}}$ to tune the polymer stiffness.

In all LJ model simulations, the time step was set to $1 \mathrm{fs}$, and the simulations for $T \leq 600 \mathrm{~K}$ were carried out 50 times with different random initial conformations for $5 \mathrm{~ns}$ each. The random initial conformations were extracted from a simulation run at a high temperature (for LJ polymers we used $400 \mathrm{~K}$ ), where the polymer essentially performs a random walk. For $T>600 \mathrm{~K}$ we did single simulations of $200 \mathrm{~ns}$. The first 500 ps were discarded for analysis. In the simulations with varying bending potential, $\left\langle S^{2}\right\rangle$ is averaged over single simulation runs of 200 ns each for every temperature.

\subsection{Atomistic Poly(3-hexylthiopene) Model}

Bhatta et al. [20] developed a force field adjusted to P3HT which we use in our work. Departing from the basic OPLS-AA force field [24], they carried out large-scale DFT calculations (B3LYP/6-31+G(d,p)) with the explicit treatment of all atoms to investigate the fully relaxed equilibrium structures of P3HT oligomers up to 10 monomer units. This approach allowed them to achieve the long-chain convergence limit for the torsional parameters. With their force field, Bhatta et al. were able to reproduce experimental findings in a bulk system such as packing structure, torsional angles of the backbone, and the hexyl side chains. The fact that this force field was designed for long chains makes it particularly suitable for our investigations. As discussed in recent in-depth force field comparisons by the authors of [25-27], many other force fields, in contrast, are designed rather for short oligomers. Here, we therefore adjusted the force field of Bhatta et al. to form long, but finite chains of P3HT by adding uncharged hydrogen atoms at the end.

Schematics showing the central part of the planar P3HT are shown in Figure 1a. Figure $1 \mathrm{~b}$ illustrates the structure of $\mathrm{P} 3 \mathrm{HT}$ in the all-atom model. The validation of the force field was done by reproducing the system mentioned in [20] and studying the torsional angle populations, which is shown in Section S2 of the supporting information.

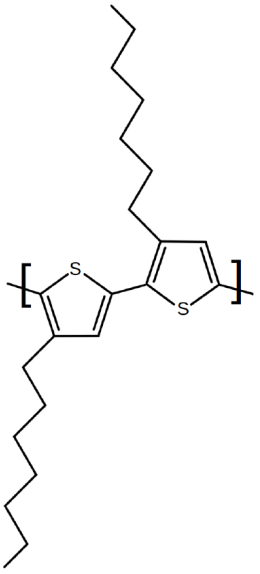

(a)

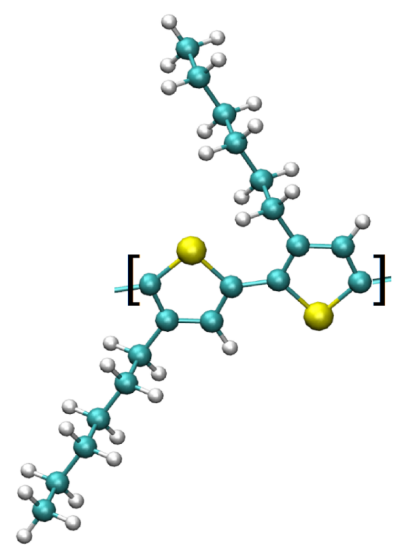

(b)

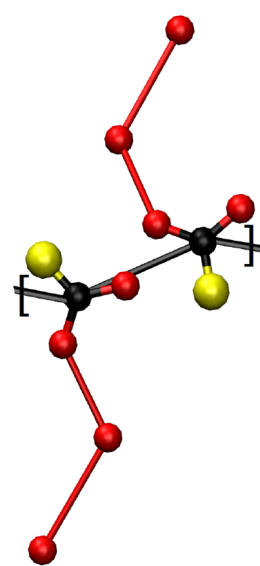

(c)

Figure 1. Schematic illustration of P3HT in (a), all-atom model in (b) and 6-site Martini CG in (c).

Similar to the LJ polymer in the previous section, the results below $600 \mathrm{~K}$ are averaged from 50 simulations of $5 \mathrm{~ns}$ each, and for above $600 \mathrm{~K}$ they are extracted from $125 \mathrm{~ns}$ NVT simulations with a timestep of $0.5 \mathrm{fs}$. Again, the first 500 ps of the simulations were 
discarded for analysis. Constraint algorithms, thermostat scaling, and periodic boundary conditions were kept the same as for the LJ polymers. Detailed results are provided in Section $\mathrm{S} 2$ of the supporting information.

\subsection{Coarse Grained Martini Poly(3-hexylthiopene) Model}

We adopted a coarse-grained (CG) model for P3HT directly from the work in [21]. The force field used follows the one created by Lee and Pao [28] and is based on the coarsegrained Martini force field [29]. The schematic representation of coarse-graining of the atomistic model with six beads per monomer is shown in Figure 1c. The distance between the two P3HT rings used in this model is $0.38 \mathrm{~nm}$. The details of the model validation can be found in [21]. Plain cut-off was used with neighborlist radius and coulomb cut-off radius set to $1.0 \mathrm{~nm}$. A velocity rescaling thermostat was used to keep the temperature constant. For temperatures below $600 \mathrm{~K}, 50 \mathrm{NVT}$ simulation runs were done for $5 \mathrm{~ns}$, and for above $600 \mathrm{~K}$ we did single simulation runs of $250 \mathrm{~ns}$ with 5 fs timestep and LINCS constraints. The initial 500 ps of the simulations were discarded when calculating the properties.

The CG Martini model of solvent tetrahydrofuran (THF) contains one bead per molecule and is taken from Patti et al. [30].

For the solvent simulations, we added 5000 THF molecules in a box of size $(78.5 \mathrm{~nm})^{3}$, pressure equilibration reduced to about $(14 \mathrm{~nm})^{3}$ at $300 \mathrm{~K}$ (further details are provided in supporting information, Section S9). We used a Nosé-Hoover thermostat with $\tau_{t}=0.4 \mathrm{ps}$ and an isotropic Parrinello-Rahman barostat with $\tau_{p}=1.0 \mathrm{ps}$ and a reference pressure of 1.0 bar. The compressibility was set to $4.5 \times 10^{-5} \mathrm{bar}^{(-1)}$ Again, for all the temperatures below $600 \mathrm{~K}$, we did 50 simulations each and the initial configurations of the polymer were extracted from the runs carried out at $2000 \mathrm{~K}$ (temperature well within the random walk limit). After the energy minimization step, we did 100 ps NVT simulation, followed by 2000 ps NPT equilibration runs. The system was then simulated for another $10 \mathrm{~ns}$ using NPT ensemble which was used for trajectory analysis. We found that $78 \mathrm{~nm}$ seems very large for $5000 \mathrm{THF}$.

\section{Results}

\section{Lennard-Jones Model Polymers}

In order to provide the necessary background for the $\mathrm{P} 3 \mathrm{HT}$ investigations to be presented below, we start with a brief study of Lennard-Jones model polymers. Following [16], we plot in Figure 2a,b the radius of gyration $\left\langle S^{2}\right\rangle$ defined in Equation (1) for a polymer with $N=200$ monomers. The maximum distance between two monomers of a polymer averaged over time $D_{\max }$ is presented in Figure 2c,d. As detailed in Section 2.2 above, the stiffness of the polymer can be tuned in two ways: in Figure 2a,c the attractive part of the LJ potential tunes the stiffness while in Figure $2 b$, $d$ we tune the bending potential.

Going from right to left in Figure 2a, stiff polymers $\left(C_{6}=0\right)$ show increasing $\left\langle S^{2}\right\rangle$ with decreasing temperature whereas flexible polymers $\left(C_{6} \geq 0.0007\right)$ show decreasing $\left\langle S^{2}\right\rangle$ with decreasing temperature. In between these two regimes, some chains $\left(C_{6}=0.0004,0.00045,0.0006\right)$ first exhibit increasing $\left\langle S^{2}\right\rangle$ with decreasing temperature followed by a sudden drop. These chains are termed semi-flexible polymers. The collapsed state minimizes the surface energy but at the cost of high bending energy. Indeed, a similar behavior is observed in Figure $2 b$, where the stiffness of the polymer is tuned by varying the bending potential. Taken together, Figure 2a,b clearly show that MD simulations confirm the intermediate swelling of the polymers before the low-temperature collapse predicted by the simpler grid-based Monte-Carlo models in [16]. The radius of gyration depending on the polymer length is compared to the classical theory of Flory [31] in Section S1 of the supporting information.

Much like in Figure 2a,b, flexible polymers show decreasing $D_{\max }$ with decreasing temperature in Figure 2c,d. Stiff polymers have increasing $D_{\max }$ with decreasing temperature whereas semiflexible polymers have increasing $D_{\max }$ with decreasing temperature followed by a drop. Thus, the maximum extension of the polymer provides an additional tool to 
separate the various conformations. It will become an important basis for further analysis of P3HT polymers.
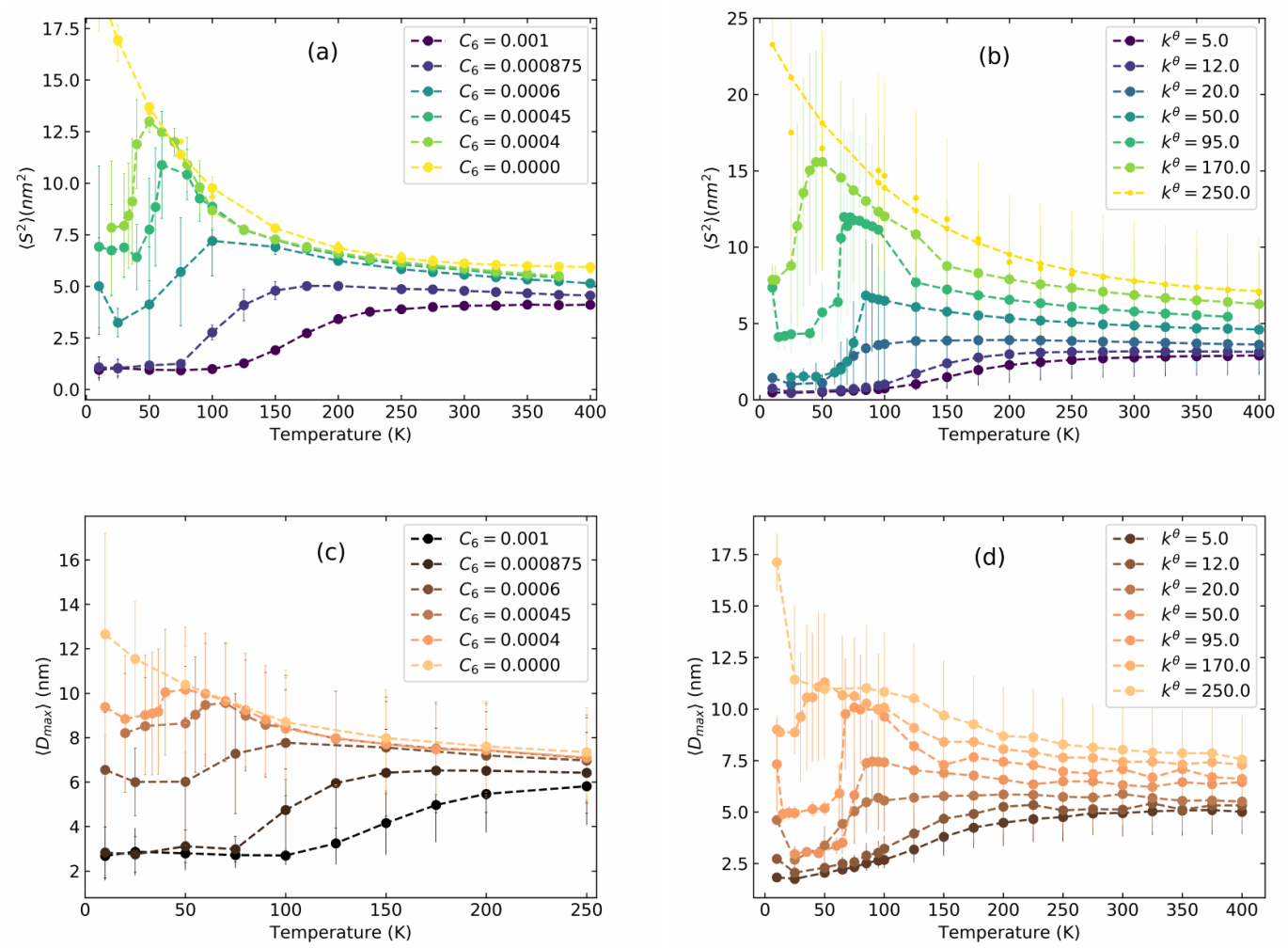

Figure 2. (a) Mean squared radius of gyration $\left\langle S^{2}\right\rangle$ as a function of temperature for a LJ polymer with $n=200$. The stiffness is tuned by the non bonded parameter $C_{6}$ in $\frac{\mathrm{kJ}}{\mathrm{mol} \mathrm{nm}^{6}}$. (b) Similar to (a), but the stiffness is tuned by varying the bending potential $k^{\theta}$ in $\frac{\mathrm{kJ}}{\mathrm{mol} \mathrm{rad}^{2}}$. (c) Average maximum distance between two monomers $D_{\max }$ as a function of temperature for an LJ polymer where the stiffness is set by $C_{6}$. (d) Similar to (c) but tuning the bending potential.

Having presented the overall nature of $D_{\max }$ and $\left\langle S^{2}\right\rangle$, we will now relate these insights to the temperature-dependent behavior for stiff, semi-flexible, and flexible polymers. As examples for these types, we select $C_{6}=0.0000$ (stiff), $C_{6}=0.00045$ (semi-flexible), and $C_{6}=0.001$ (flexible). Figure 3a represents the histograms for $\left\langle S^{2}\right\rangle$ and Figure $3 \mathrm{~b}$ represents the histograms for $D_{\max }$ at different temperatures.

The names of the structures that will now be used for further discussion are shown in Figure $4 \mathrm{a}$, which illustrates the different forms attained by the polymer. Going from left to right, the compact circularly wound form is labeled as a toroid (A), the multiple folded elongated structure is a bundle (B), the random coil is (C), a single circularly wound form is termed a ring (D), and finally a single folded elongated structure is termed a hairpin (E). The corresponding colors are light orange, dark orange, yellow, dark blue, and light blue, respectively. The nomenclature of these conformations is based on various previous studies [18,32-34] with whom we compare in Section S3 of the supporting information.

Starting with the distributions for the stiff polymer in Figure $3 a$, we see a peak at $5 \mathrm{~nm}^{2}$ at the highest temperature $(250 \mathrm{~K})$ with a tail towards higher radii. This structure corresponds to a random open coil that opens and closes rapidly and is thus marked with the label C. As we lower the temperature from $250 \mathrm{~K}$ to $100 \mathrm{~K}$ and further down to $75 \mathrm{~K}$, we see a shift towards higher radii and a broadening of the distribution. At the lowest temperature of $10 \mathrm{~K}$, a clear second peak at about $28 \mathrm{~nm}^{2}$ has appeared which corresponds to a stiffened and more elongated coil. This transition from rapidly opening and closing random coil to a more rigid coil gives rise to the an exponential increase in the $\left\langle S^{2}\right\rangle$ curve 
in Figure $2 \mathrm{a}, \mathrm{b}$ for stiff polymers. The maximum extension exhibits a very similar behavior as shown in Figure 3d.
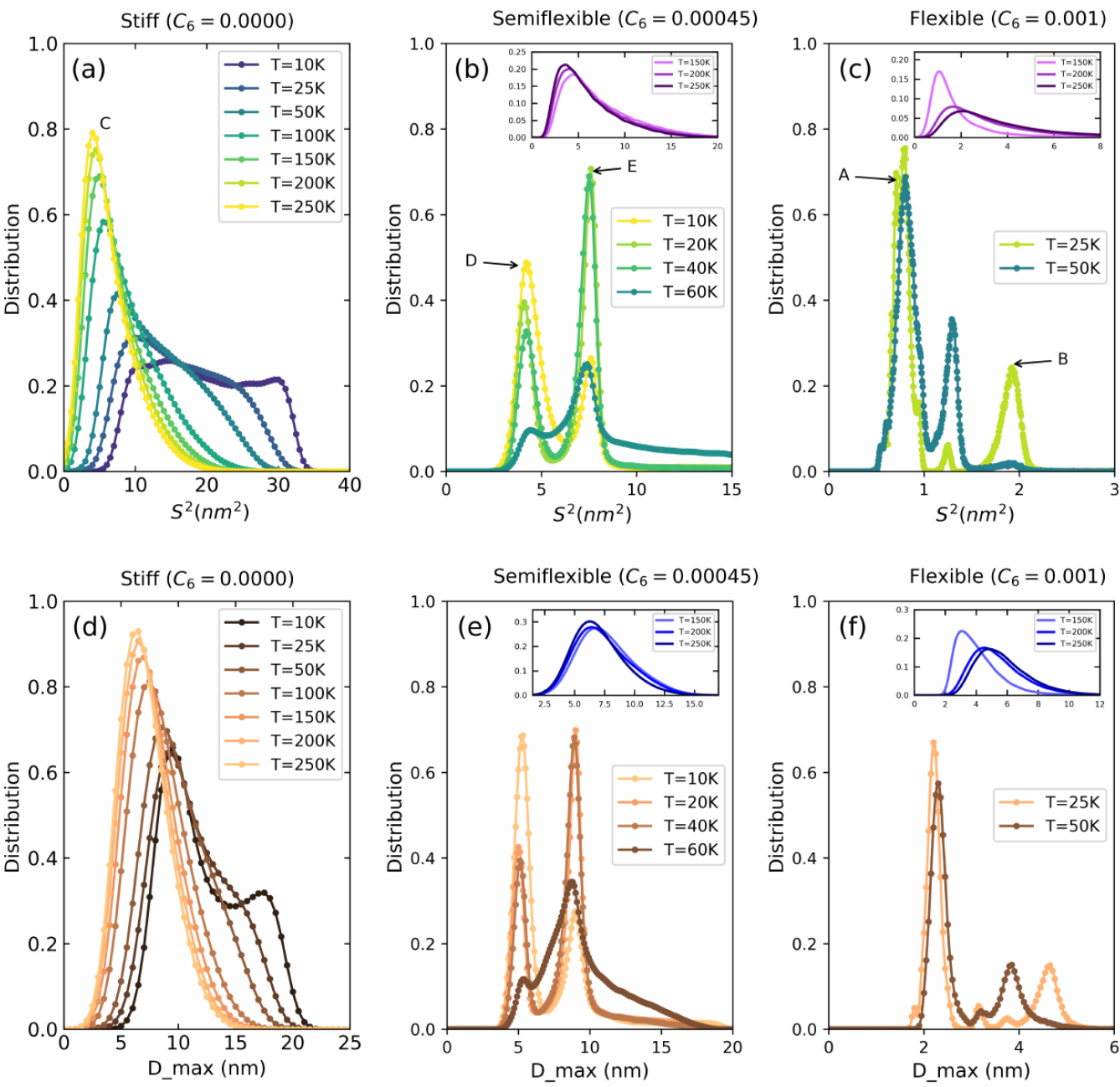

Figure 3. Distribution of $S^{2}$ for (a) stiff $\left(C_{6}=0.0000\right),(\mathbf{b})$ semiflexible $\left(C_{6}=0.00045\right)$, and (c) flexible $\left(C_{6}=0.001\right)$ polymers. Distribution of $D_{\max }(\mathbf{d})$ stiff $\left(C_{6}=0.0000\right),(\mathbf{e})$ semiflexible $\left(C_{6}=0.00045\right)$, and (f) flexible $\left(C_{6}=0.001\right)$ polymers.

Semiflexible polymers are exceptionally interesting because of their swelling-beforecollapse transition. When going from higher to lower temperatures, semiflexible polymers at first exhibit an increase in $\left\langle S^{2}\right\rangle$ due to a stiffening of the coil similar to the stiff polymers. However, at a certain temperature $(50 \mathrm{~K}<T<75 \mathrm{~K})$ a collapse to either hairpins or rings is observed.

To study this collapse in more detail, Figure $3 \mathrm{~b}$ shows the distribution first at temperatures below the collapse. At $10 \mathrm{~K}$, this allows us to identify two clear peaks at $4.1 \mathrm{~nm}^{2}$ and $7.8 \mathrm{~nm}^{2}$ marked with the labels D and E, respectively. These confirm that the probability of forming rings and hairpins exists at all temperatures. As we increase the temperature from $10 \mathrm{~K}$ to $60 \mathrm{~K}$, the peak at $4.1 \mathrm{~nm}^{2}$ decreases and the one at $7.8 \mathrm{~nm}^{2}$ increases indicating a shift in probability of the polymer for forming rings at low temperature $(10 \mathrm{~K})$ and hairpins at relatively higher temperatures $(60 \mathrm{~K})$. Above $70 \mathrm{~K}$, the polymer forms an open random coil with a wide $\left\langle S^{2}\right\rangle$ distribution as shown in the inset of Figure $3 \mathrm{~b}$. Again, the $D_{\max }$ distribution in Figure 3e exactly mirrors this behavior.

Flexible polymers shown in Figure 3c, in general, behave similarly to semiflexible polymers, however, their interplay at lower temperatures is between a toroid and a bundle rather than a ring and a hairpin. Therefore, the values of $\left\langle S^{2}\right\rangle$ are in general smaller. 
For temperatures above $150 \mathrm{~K}$, we again observe a wide distribution corresponding to the random open coil conformation. At low temperatures $(10-50 \mathrm{~K})$ the peaks appear at $0.8 \mathrm{~nm}^{2}$ (marked with label A for toroids), $1.2 \mathrm{~nm}^{2}$, and $2.0 \mathrm{~nm}^{2}$ (marked with label B for bundles) which indicates the existence of both toroids and bundles as well as an unstable state between the two. Quantitalively similar behavior is reproduced by the histograms of $D_{\max }$ in Figure $3 \mathrm{f}$.

As suggested by the $\left\langle S^{2}\right\rangle$ distributions, the final conformation that a polymer attains depends on its initial conformation. In order to properly account for this behavior, multiple simulations with different initial conditions were carried out for each set of parameters. By visual inspection and comparison to the generic structures in Figure 4a, we then determined the type of conformation for each simulation. Figure $4 \mathrm{~b}$ shows color coded final conformations for 50 simulations at each $C_{6}$ value and temperature. This allows visualizing the transition from flexible to semiflexible to stiff polymers by the gradual change from orange to blue to yellow region, respectively.

Furthermore, it allows us to study the $D_{\max }$ distributions within each conformation. For this, we combine all conformations of a given type (A-E) independent of their $C_{6}$ and temperature and plot the corresponding distributions in Figure 4c. Clearly, the toroid is the most compact form with a $D_{\max }$ peak at $2 \mathrm{~nm}$, followed by the bundle at $3.75 \mathrm{~nm}$, the ring at $5.3 \mathrm{~nm}$, and the hairpin at $8.8 \mathrm{~nm}$. The random coil is a very unstable structure with $D_{\text {max }}$ ranging from 7.5 to $20 \mathrm{~nm}$.

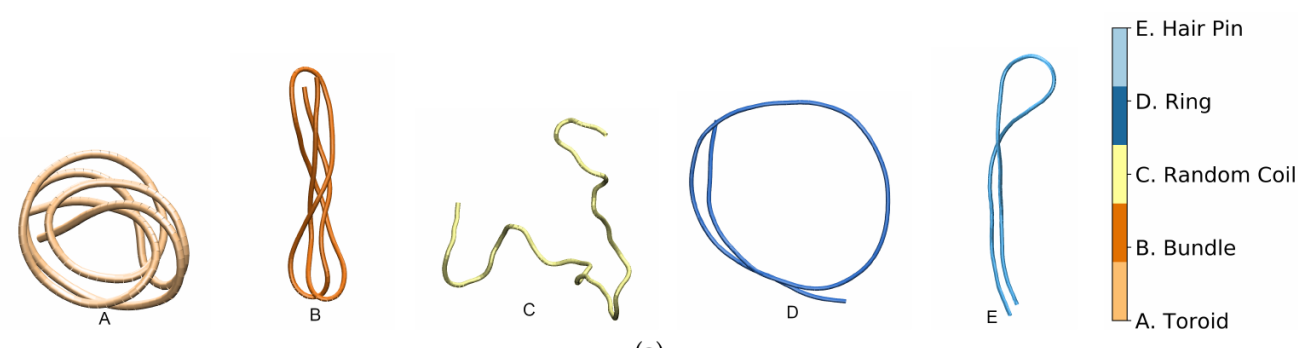

(a)

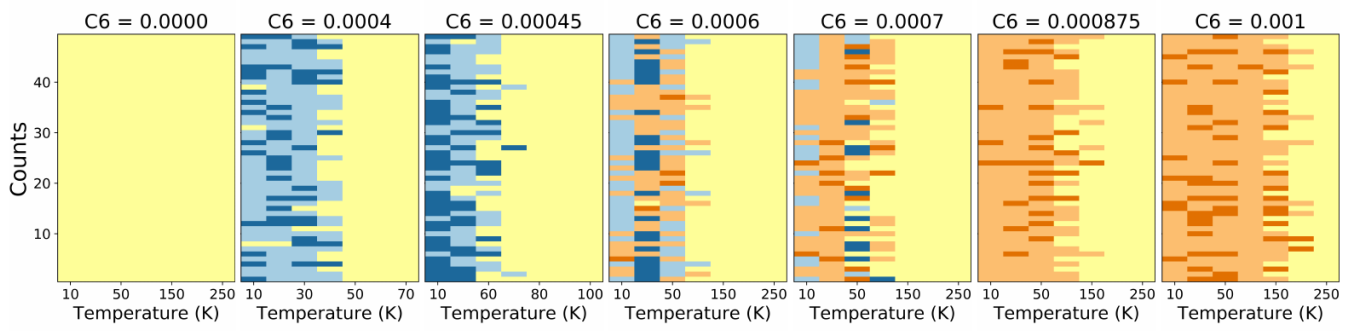

(b)

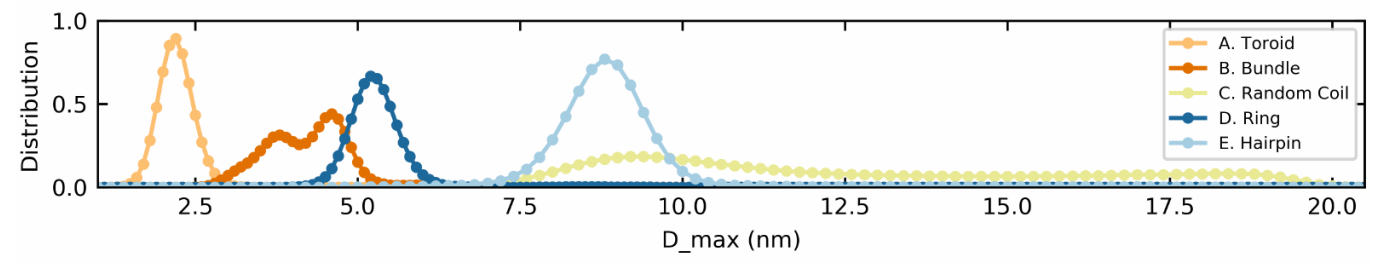

(c)

Figure 4. (a) From left to right illustration of toroid, bundle, random coil, ring and hairpin. (b) Color coded final conformations at a range of temperature for 50 simulations at different $C_{6}$ values. (c) $D_{\max }$ distribution of the above five mentioned structures.

\section{Poly(3-hexylthiopene) (P3HT)}

\subsection{Atomistic P3HT}

We now turn to study a realistic, atomistically resolved model for a single P3HT chain with 200 monomers. In this subsection, simulations are carried out in a vacuum mimicking a very bad solvent as detailed in Section 2.3. 
Figure 5a shows the averaged $D_{\max }$ at different temperatures. Most striking is the sigmoidal increase of $D_{\max }$ with temperature which clearly reminds one of the LJ polymers in Figure 2. We note, however, that the sigmoidal increase happens at fairly high temperatures which are not usually accessible in experiments, as P3HT typically fully decomposes for temperatures above around $850 \mathrm{~K}$ [35]. In order to make the connection to experimental observations later, we, therefore, focus on the temperature range at and below $600 \mathrm{~K}$ which is enhanced in the inset. Interestingly, we here observe a slight but systematic swelling of the structure demonstrated by an increase in $D_{\max }$ towards lower temperatures. To investigate this phenomenon further, we revert to the machinery established above for the LJ polymers and show in Figure $5 \mathrm{~b}$ the $D_{\max }$ distributions at different temperatures. At low temperatures, three clear peaks are observed. Visual inspection allows us to identify the left-most peak at about $8 \mathrm{~nm}$ with a toroid structure and the right-most peak at about $12.5 \mathrm{~nm}$ with a bundle. The corresponding conformations are shown in Figure $5 \mathrm{c}$. In contrast to the LJ model polymer of Figure 3b, however, P3HT exhibits a prominent third peak at intermediate $D_{\max }$ around $11 \mathrm{~nm}$. This peak corresponds to a tight globular structure for which an example is also shown in Figure 5c. A more extensive set of conformations for these conformations is provided in Section S5 of the supporting information while similar data for $\left\langle S^{2}\right\rangle$ is shown in supporting information Section S4.1.

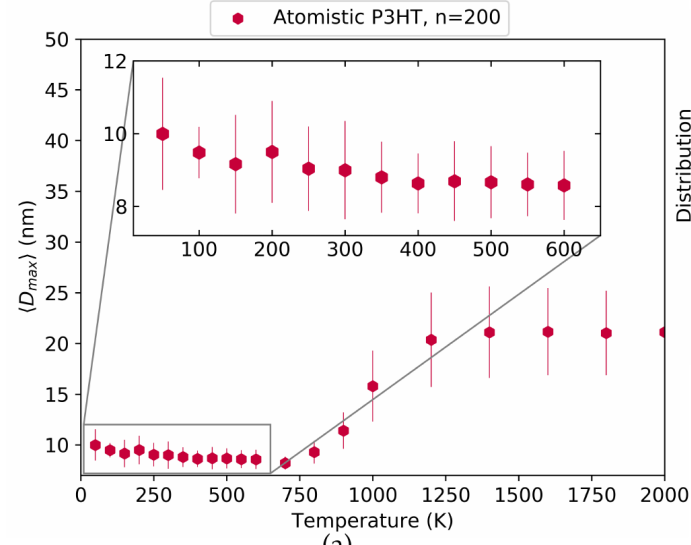

(a)

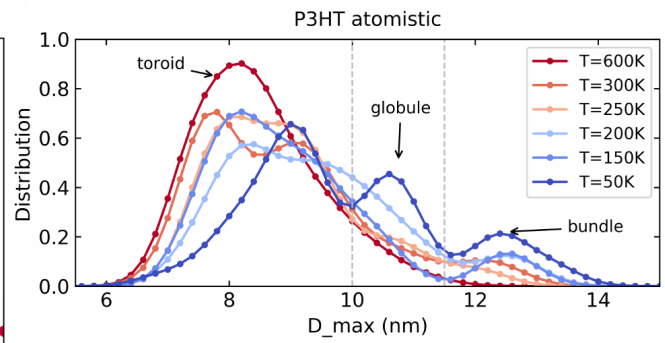

(b)
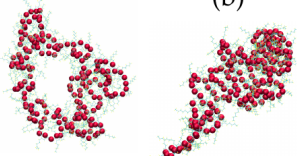

(c)

Figure 5. The maximum distance between monomers for the atomistic P3HT model in vacuum at different temperatures. (a) The averaged $D_{\max }$ as a function of temperature shows a sigmoidal increase at very high and a slight increase towards low temperatures (inset). (b) The $D_{\max }$ distributions at different temperatures exhibit three clear peaks corresponding to toroids, tight coils and bundles (from left to right) which are illustrated in (c).

Similar to the LJ polymers, we run a set of 50 simulations with different starting conditions for each temperature. Using $D_{\max }$ from the final simulation frame and comparing to the dashed lines in Figure 5b allows us to classify the resulting structures into toroid, tight globule, and bundle. The result is shown in Figure 6a. Toroids are marked by pink and lie in the range of $0 \mathrm{~nm}<D_{\max }<10.0 \mathrm{~nm}$, globules are marked by grey and have $10.0 \mathrm{~nm}<D_{\max }<11.5 \mathrm{~nm}$ while bundles have $D_{\max }>11.5 \mathrm{~nm}$ and are marked by red. As we decrease the temperature from $600 \mathrm{~K}$ to $50 \mathrm{~K}$, the bundle conformations clearly become more frequent. However, for most of the temperatures below $300 \mathrm{~K}$, both bundle and toroid structures coexist along with tight globule. Picking out all the simulations in the toroid, tight globule, and bundle regions individually we plot the $D_{\max }$ distribution averaged over last 500 ps of each run in Figure $6 \mathrm{~b}$ which underscores how each conformation directly corresponds to a distinct peak of $D_{\max }$. in [25].

Finally, we note that we have not observed helical structures such as those shown 


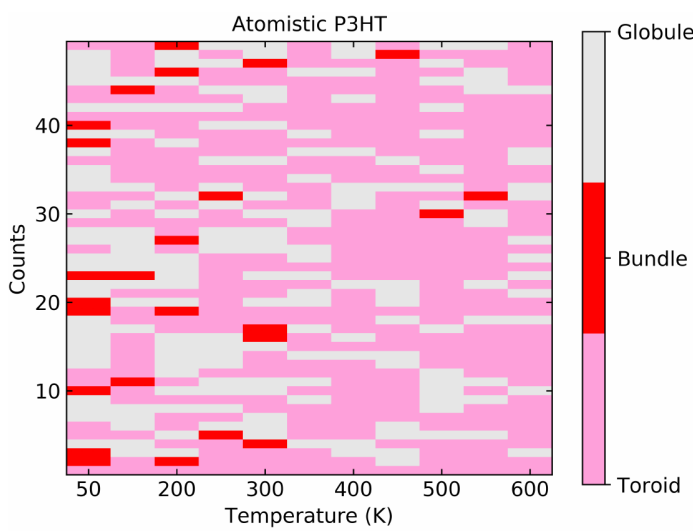

(a)

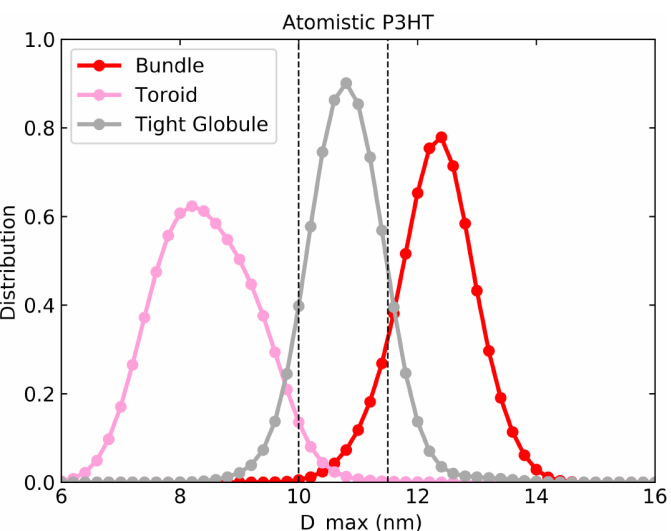

(b)

Figure 6. (a) Color coded final conformations at a range of temperature for 50 simulations for atomistic P3HT in vacuum. (b) $D_{\max }$ distributions used for toroid, globules, and bundles.

\subsection{Martini P3HT in Vacuum}

We now proceed with a similar analysis for our coarse-grained Martini model of P3HT, again starting in vacuum and using $n=200$. As detailed in Section 2.4, here each P3HT monomer is described by six beads.

Figure 7a shows averaged $D_{\max }$ while Figure $7 \mathrm{~b}$ displays the $D_{\max }$ distributions at the different temperatures similar to Figure 5 above. In Figure 7 a we again see a simple sigmoidal increase in $D_{\max }$ for very high temperatures and a less pronounced, but still systematic swelling for decreasing temperatures within a realistic range of $600 \mathrm{~K}$ to $100 \mathrm{~K}$. The $D_{\max }$ distribution in Figure $7 \mathrm{~b}$ is in good agreement with the atomistic model shown in Figure 5 above.

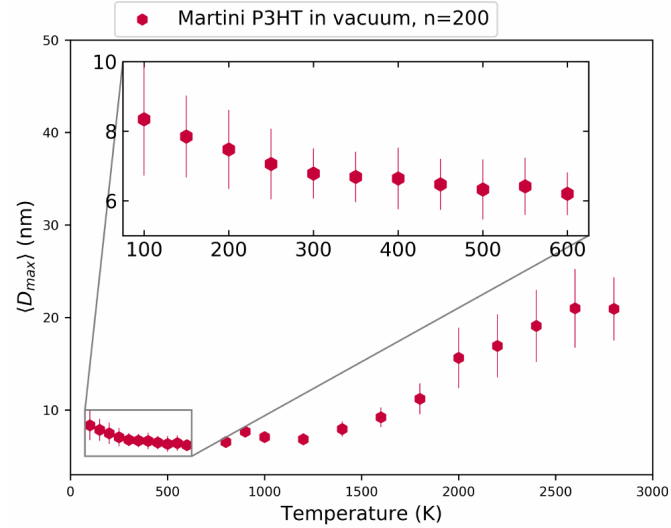

(a)

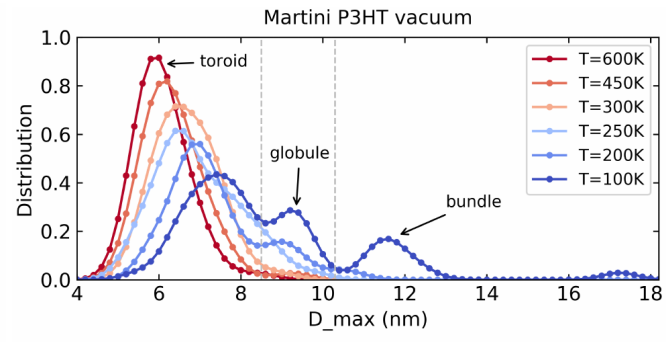

(b)

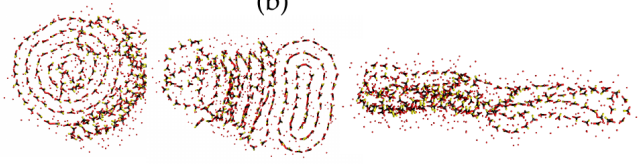

(c)

Figure 7. The maximum distance between monomers for the Martini model of P3HT in vacuum. (a) Averaged $D_{\max }$ as a function of temperature. (b) Distributions at various temperatures. (c) Illustration of the corresponding conformations: toroids, tight globules and bundles.

We notice three prominent peaks at low and a single strong peak at high temperatures. The transition between these peaks upon a change in temperature proceeds in a similar fashion as for the atomistic case with the only difference being that the Martini model overall exhibits slightly more compact structures than the atomistic model. Visual inspection of the corresponding real-space conformations allows identifying again the sequence of toroids, tight globules, and bundles as shown in Figure 7c. The borders between these phase as marked by the grey lines in Figure $7 \mathrm{~b}$ are $0 \mathrm{~nm}<D_{\max }<8.5 \mathrm{~nm}$ for toroids, $8.5 \mathrm{~nm}<D_{\max }<10.3 \mathrm{~nm}$ for tight globules and finally $D_{\max }>10.3 \mathrm{~nm}$ for bundles. In fact, the geometric characteristics are even more pronounced than in the atomistic model. For additional conformations and $\left\langle S^{2}\right\rangle$ data, see supporting information, Sections S4.2 and S6. 
Figure 8a represents the color-coded outcome of the last frame of the 50 simulations run at each temperature with different initial conditions. Figure $8 b$ shows the individual distribution of $D_{\max }$ for toroids, globules, and bundles. Not surprisingly, both figures show very good agreement with the atomistic model in Figure 6.

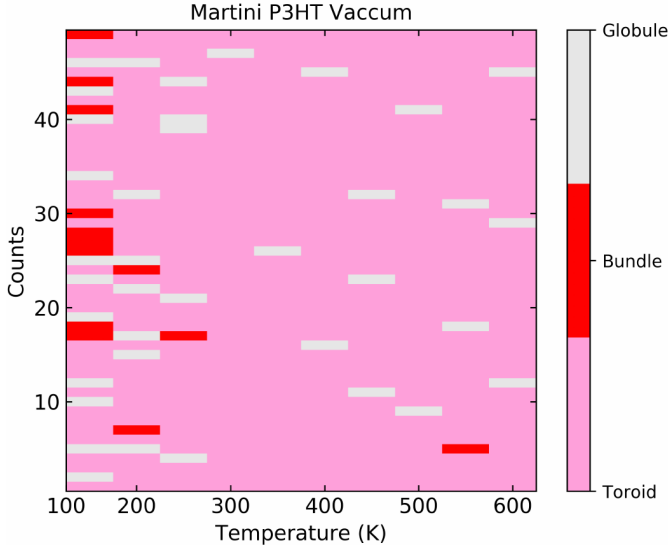

(a)

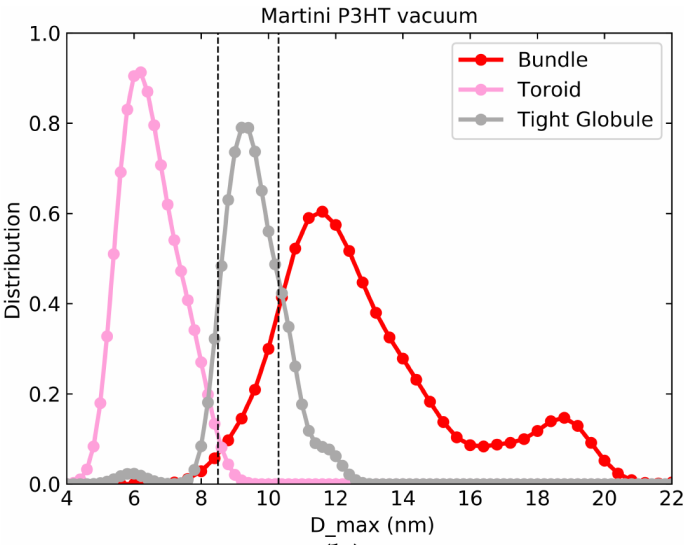

(b)

Figure 8. (a) Color coded final conformations at a range of temperature for 50 simulations for the Martini model of P3HT in a vacuum. (b) $D_{\max }$ distributions used for toroid, globules, and bundles.

\subsection{Martini P3HT in THF Solvent}

In order to match more closely a typical experimental situation such as the one in $[14,15]$, we now study the behavior of P3HT chains in THF solvent. For the solvent, we use the Martini model described in the Methods Section 2.4 above. The maximum extension as a function of temperature shown in Figure 9a is similar to the previous cases in a vacuum with one notable exception: at about $220 \mathrm{~K}$ we observe a rather abrupt increase in $D_{\max }$ (going from right to left) after which the values decrease again. This corresponds to a swelling of the molecule and is also reflected, albeit less strongly, in the radius of gyration shown in $\mathrm{S} 4.3$ of the supporting information.

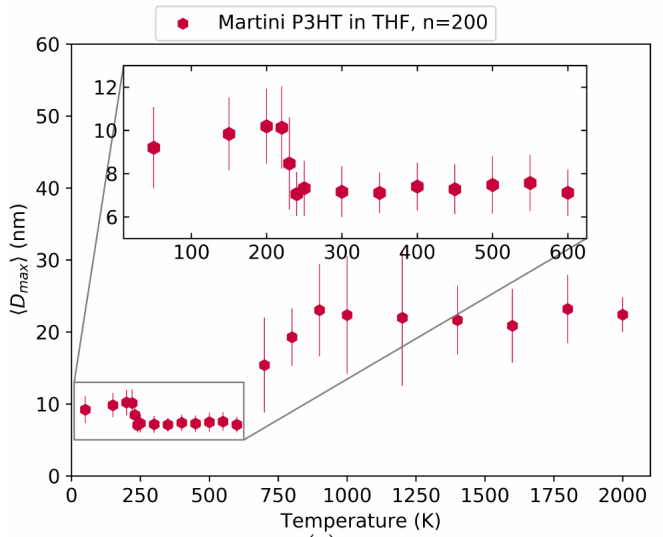

(a)

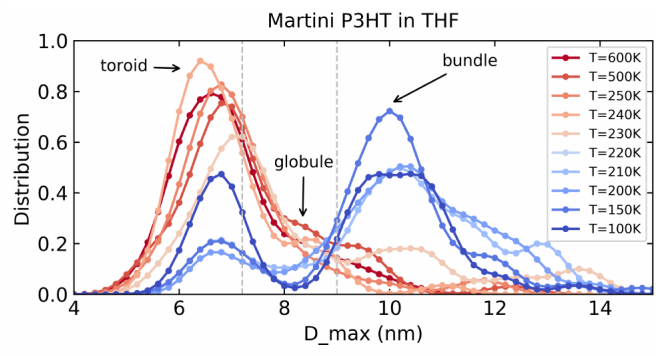

(b)

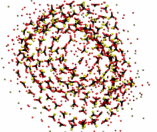

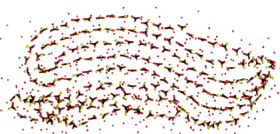

(c)

Figure 9. The maximum distance between monomers for the Martini model of P3HT in THF solvent. (a) Averaged $D_{\max }$ as a function of temperature. (b) Distributions at various temperatures. (c) Illustration of the corresponding conformations: toroids, tight globules and bundles.

Looking at the distributions in Figure 9b, we note as a further difference that the middle peak is no longer present. Nevertheless, the two prominent peaks at around $6 \mathrm{~nm}$ and $10 \mathrm{~nm}$ corresponding to toroids and bundles, respectively, are still clearly visible. The geometrical appearance of toroids and bundles is in general similar to the vacuum case as seen by the examples in Figure $9 \mathrm{c}$ as well as Section S7 of the supporting information. In 
Figure 10 we show the occurrence probabilities of toroid, bundles and globules for the 50 different initial conditions. Similar to the above two cases, the criteria for $D_{\max }$ that was used for distinguishing these classes is shown by dashed gray lines in Figure 9b. Toroids lie in the range of $0<D_{\max } \leq 7.2 \mathrm{~nm}$, globules within $7.2<D_{\max } \leq 9 \mathrm{~nm}$, and bundles have dimensions $D_{\max }>9 \mathrm{~nm}$. We note that one effect of the solvent is to increase the frequency of the bundle as compared to the vacuum case. As confirmed by the larger size of the bundle structure (see Figure 9b), this explains the sudden rise in $D_{\max }$ at around $220 \mathrm{~K}$.

As the situation in THF is the most experimentally relevant, we provide here two further characterizations of the observed structures: the $\pi$ - $\pi$-stacking distance and the conjugation length. We compute the $\pi$ - $\pi$-stacking distance within the bundle structure in which the chains fold back multiple times on themselves as clearly seen in Figure 9c. This backfolding is particularly important as it is connected to the electronic mobility in organic transistors by virtue of the $\pi-\pi$ stacks. Following the analysis methods of [12], we found that the distance between the $\pi-\pi$ stacked layers in our bundle conformations amounts to $4.73 \pm 0.23 \mathrm{~nm}$. The slight deviation of this number from the experimental value may be attributed to the approximation of the thiophene ring as a spherical site in the CG model, which may overestimate the steric bulk of the ring in the $\pi-\pi$ direction.

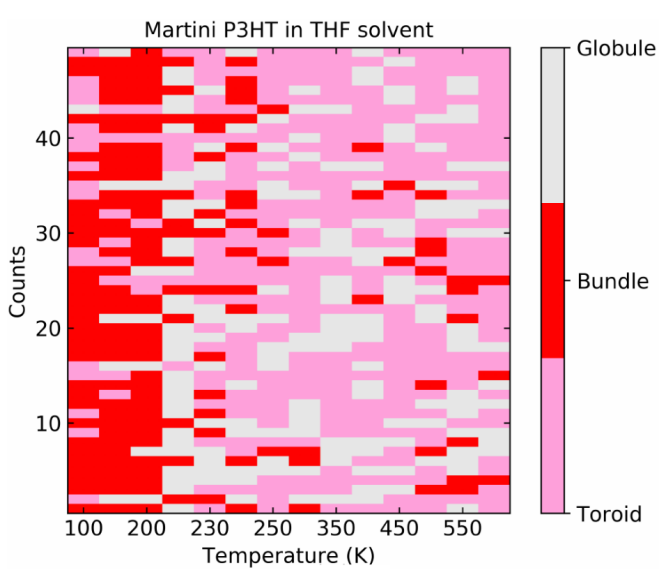

(a)

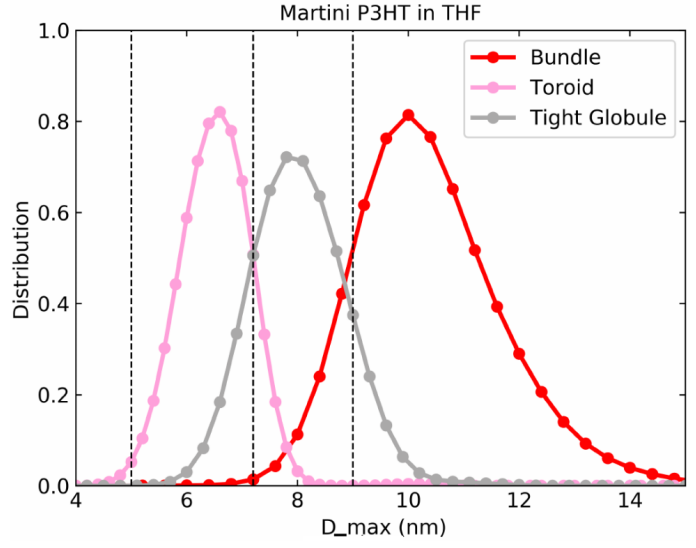

(b)

Figure 10. (a) Color coded final conformations at a range of temperature for 50 simulations for the Martini model of P3HT in THF solvent. (b) $D_{\max }$ distributions used for toroid, globules and bundles.

The conjugation length is an intrinsically electronic quantity, however, a first estimate can be obtained from the distribution of dihedral angles. For this, we assume that P3HT is broken into electronically isolated units (chromophores) if the inter-monomer dihedral angle exceeds a particular threshold value. Following previous estimates of conjugation lengths in simulations of P3HT [36,37] a threshold value of $40^{\circ}$ was chosen. The conjugation length is then given by the number of consecutive monomers whose angle lies below the threshold.

The calculated distributions of conjugation lengths for bundles and toroids are shown in Figure 11. We find that large conjugation lengths in bundles are more than twice as likely as in toroids, which clearly demonstrates increased planarization of the polymer chains below $220 \mathrm{~K}$. 


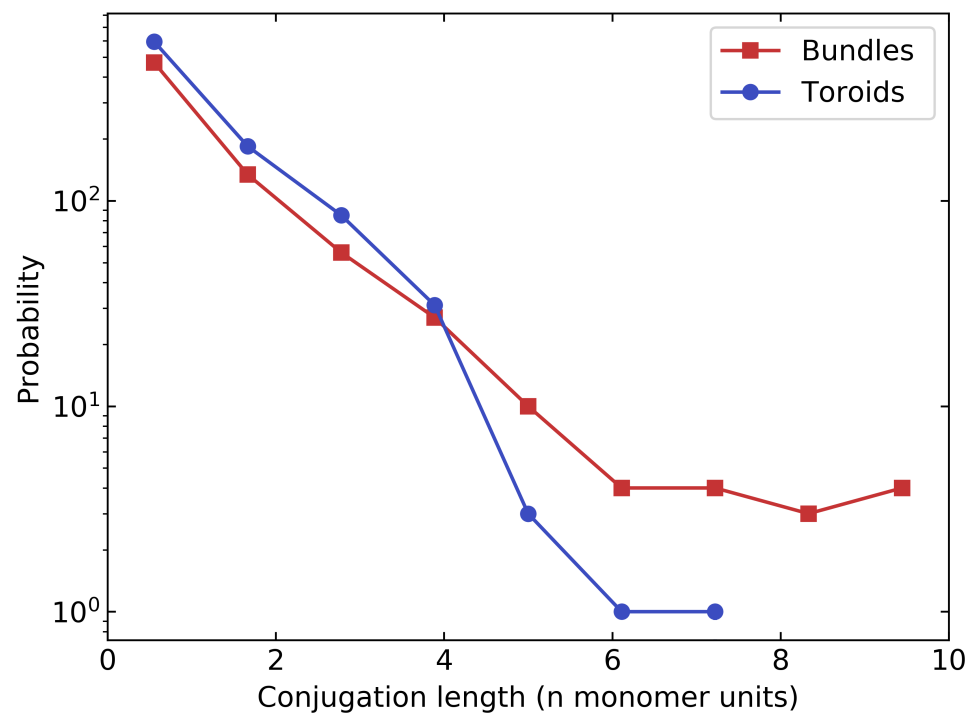

Figure 11. Distribution of conjugation lengths in toroids and bundles for P3HT in THF solvent.

\section{Discussion and Conclusions}

In summary, our detailed comparison shows that $\mathrm{P} 3 \mathrm{HT}$ in vacuum inherits various typical characteristics of flexible polymers, namely the sigmoidal increase in $\left\langle S^{2}\right\rangle$ and $D_{\max }$ upon increasing the temperature and the occurrence of toroids and bundles at low temperatures. Once immersed into THF solvent, however, new phenomena arise. Most prominently, we observe a rather sharp swelling of the molecule at a temperature of around $220 \mathrm{~K}$.

This swelling behavior is indeed reflected closely in the recent series of spectroscopic experiments by Panzer et al. $[14,15,38]$. Panzer and coworkers worked on P3HT with molecular weights ranging from $5 \mathrm{kDa}$ to $34 \mathrm{kDa}$ corresponding to 30 to 200 monomers per polymer where they observed a change from red to blue in the absorption as well as emission spectra as they decreased the temperature. The spectroscopic signatures were interpreted that the polymer takes a random coil conformation at high temperatures and at lower energies the backbone and also the side chains planarize to form a crystalline planar structure which they assumed to be a "planarized swollen coil". The change in the absorption spectra occurred via a bathochromic shift, suggesting an increased conjugation length which Panzer et al. connected to a swelling of the polymer before the final collapse into an ordered state (planarized) state. We hypothesize that this swelling is indeed connected to the rise of $D_{\max }$ at $220 \mathrm{~K}$ in Figure 9a and to the increased conjugation length of bundle structures in Figure 11. Our simulations then furnish further insight into this structure which indeed is swollen (in terms of an increase in $D_{\max }$ ) and in addition assumes a planar, bundle-like structure as shown in Figure 9c. The simulations thus allow us to interpret the swelling as an increased frequency of bundle structures that are more elongated than the compact globule and toroid structures observed at higher temperatures.

To check the robustness of the observed effect, we conducted simulations with chain lengths of $n=112$ and $n=68$. $D_{\max }$ as a function of temperature for these chains is shown in Figure 12. The transition temperature at which the swelling takes place is clearly visible around $185 \mathrm{~K}$ for $n=112$ while it is not so clearly distinguishable for the very short $n=68$ chain. This is in agreement with reports from the experiments in [15] that the critical temperature of P3HT chains in THF solvent decreases with decreasing chain length. Additional information about the distribution of $D_{\max }$ is provided in Section $\mathrm{S} 8$ of the supporting information. 


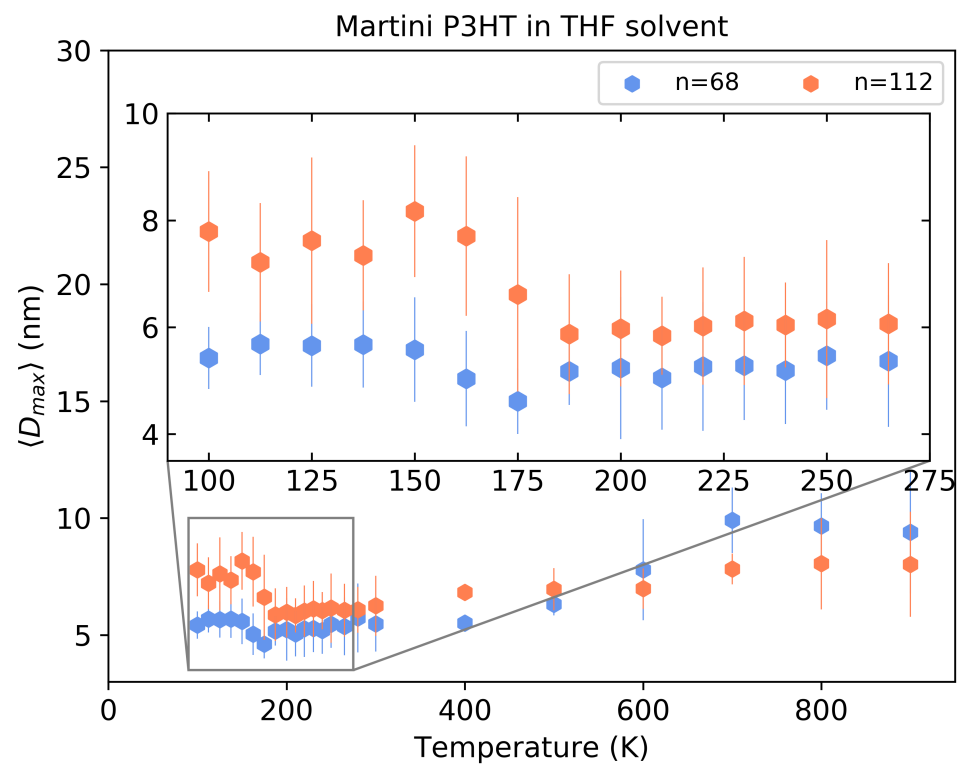

Figure 12. The maximum distance between monomers for the Martini model of P3HT with $n=68$ and $n=112$ in THF solvent.

Interestingly, our simulations predict that this swelling is closely connected to the presence of the solvent THF which might make it a worthwhile effort to study the same transition in different in the future.

Supplementary Materials: The following are available online at https:/ / www.mdpi.com/article/ $10.3390 /$ polym14030550/s1. Figure S1: Scaling of the mean-squared radius of gyration with the monomer number $\mathrm{n}$ shows ideal random walk behavior at high temperatures for the LJ model polymer. Figure S2: Snapshot of simulation box containing P3HT with 30 monomers and 32 repeats. (a) Side view with P3HT side chains going in and out of the plane. (b) Top view along the length of P3HT. (c) $\pi-\pi$ stacking along with P3HT stacking along y-axis (d) Schematic of P3HT central portion. Figure S3: Torsional population at 300K for P3HT chains with $n=13,20,30$ as a function of (a) $\alpha$, (b) $\beta_{1}$ and (c) $\beta_{2}$ which show highest populations approximately at 180, 90 and 180 degrees respectively. Figure S4: Structural phase diagrams with respect to bending constant of our LJ model system. The conformation labels here follow the notation of Zierenberg et al (2016), and thus deviate from the ones used in the main text: R(random coil), H (hairpin), D3 (rod like bundle), K (toroids), C (globular). Figure S5: The state diagram of semiflexible polymers for chain stiffness (bending potential) as a function of inverse of temperature obtained from our MD simulations of an LJ model polymer. Figure S6: For Atomistic P3HT with $\mathrm{n}=200:(\mathbf{a})\left\langle S^{2}\right\rangle$ as a function of temperature. (b) $S^{2}$ distribution as a function of temperature. This figure corresponds to the data of figure 5 of the paper. Figure S7: For Martini CG P3HT in vacuum with $n=200:(a)\left\langle S^{2}\right\rangle$ as a function of temperature. (b) $S^{2}$ distribution as a function of temperature. This figure corresponds to the data of figure 7 of the paper. Figure S8: For Martini CG P3HT in THF solvent with $n=200:(a)\left\langle S^{2}\right\rangle$ i as a function of temperature. (b) $S^{2}$ distribution as a function of temperature. This figure corresponds to the data of figure 9 of the paper. Figure S9: Typical toroids, globules and bundles found in the atomistic P3HT simulations at 300K. Figure S10: Typical toroids, globules and bundles found in the martini CG P3HT vacuum simulations at 200K-250K. Figure S11: Typical toroids, globules and bundles found in the martini CG P3HT in THF solvent at 300K. Figure S12: $D_{m}$ ax Distribution for different temperatures of Martini CG P3HT in THF solvent with (a) $n=68$ and (b) $n=112$. Figure S13: Box length for different simulations at $300 \mathrm{~K}$.

Author Contributions: Conceptualization, S.P. and S.G.; methodology, S.P. and S.G.; validation, S.P.; writing-original draft preparation, S.P. and S.G.; writing-review and editing, S.P. and S.G.; supervision, S.G.; project administration, S.G.; funding acquisition, S.G. All authors have read and agreed to the published version of the manuscript. 
Funding: We thank the Volkswagen Foundation for financial support. This publication was funded by the University of Bayreuth Open Access Publishing Fund.

Institutional Review Board Statement: Not applicable.

Informed Consent Statement: Not applicable.

Data Availability Statement: Data is available upon request.

Acknowledgments: S.P. acknowledges support by the study program "Biological Physics" of the Elite Network of Bavaria.

Conflicts of Interest: The authors declare no conflict of interest.

\author{
Abbreviations \\ The following abbreviations are used in this manuscript: \\ P3HT Poly(3-hexylthiopene) \\ THF Tetrahydrofuran \\ MD Molecular Dynamics \\ ATB Automated Topology Builder and Repository
}

\title{
References
}

1. Poelking, C.; Andrienko, D. Effect of Polymorphism, Regioregularity and Paracrystallinity on Charge Transport in Poly(3hexylthiophene) [P3HT] Nanofibers. Macromolecules 2013, 46, 8941-8956. [CrossRef]

2. Bao, Z.; Dodabalapur, A.; Lovinger, A.J. Soluble and processable regioregular poly(3-hexylthiophene) for thin film field-effect transistor applications with high mobility. Appl. Phys. Lett. 1996, 69, 4108-4110. [CrossRef]

3. Yang, H.; LeFevre, S.W.; Ryu, C.Y.; Bao, Z. Solubility-driven thin film structures of regioregular poly(3-hexyl thiophene) using volatile solvents. Appl. Phys. Lett. 2007, 90, 172116. [CrossRef]

4. Goh, C.; Kline, R.J.; McGehee, M.D.; Kadnikova, E.N.; Fréchet, J.M.J. Molecular-weight-dependent mobilities in regioregular poly(3-hexyl-thiophene) diodes. Appl. Phys. Lett. 2005, 86, 122110. [CrossRef]

5. Kuila, B.K.; Nandi, A.K. Structural Hierarchy in Melt-Processed Poly(3-hexyl thiophene)-Montmorillonite Clay Nanocomposites: Novel Physical, Mechanical, Optical, and Conductivity Properties. J. Phys. Chem. B 2006, 110, 1621-1631. [CrossRef]

6. Heffner, G.W.; Pearson, D.S. Molecular characterization of poly(3-hexylthiophene). Macromolecules 1991, 24, 6295-6299. [CrossRef]

7. McCulloch, B.; Ho, V.; Hoarfrost, M.; Stanley, C.; Do, C.; Heller, W.T.; Segalman, R.A. Polymer Chain Shape of Poly(3alkylthiophenes) in Solution Using Small-Angle Neutron Scattering. Macromolecules 2013, 46, 1899-1907. [CrossRef]

8. Nagai, M.; Huang, J.; Zhou, T.; Huang, W. Effect of molecular weight on conformational characteristics of poly(3-hexyl thiophene). J. Polym. Sci. Part B Polym. Phys. 2017, 55, 1273-1277. [CrossRef]

9. Adachi, T.; Brazard, J.; Ono, R.J.; Hanson, B.; Traub, M.C.; Wu, Z.Q.; Li, Z.; Bolinger, J.C.; Ganesan, V.; Bielawski, C.W.; et al. Regioregularity and Single Polythiophene Chain Conformation. J. Phys. Chem. Lett. 2011, 2, 1400-1404. [CrossRef]

10. Chen, P.Y.; Rassamesard, A.; Chen, H.L.; Macromolecules, S.C. Conformation and fluorescence property of poly (3-hexylthiophene) isolated chains studied by single molecule spectroscopy: Effects of solvent quality and Regioregularity. ACS Publ. 2013, 46, 56575663. [CrossRef]

11. Raithel, D.; Baderschneider, S.; Queiroz, T.B.d.; Lohwasser, R.; Köhler, J.; Thelakkat, M.; Kümmel, S.; Hildner, R. Emitting Species of Poly(3-hexylthiophene): From Single, Isolated Chains to Bulk. Macromolecules 2016, 49, 9553-9560. [CrossRef]

12. Schwarz, K.N.; Kee, T.W.; Huang, D.M. Coarse-grained simulations of the solution-phase self-assembly of poly(3-hexylthiophene) nanostructures. Nanoscale 2013, 5, 2017-2027. [CrossRef]

13. Tapping, P.C.; Clafton, S.N.; Schwarz, K.N.; Kee, T.W.; Huang, D.M. Molecular-Level Details of Morphology-Dependent Exciton Migration in Poly(3-hexylthiophene) Nanostructures. J. Phys. Chem. C 2015, 119, 7047-7059. [CrossRef]

14. Panzer, F.; Baessler, H.; Lohwasser, R.; Thelakkat, M.; Keohler, A. The impact of polydispersity and molecular weight on the order disorder transition in poly (3-hexylthiophene). J. Phys. Chem. Lett. 2014, 5, 2742-2747. [CrossRef]

15. Panzer, F.; Baessler, H.; Koehler, A. Temperature induced order-disorder transition in solutions of conjugated polymers probed by optical spectroscopy. J. Phys. Chem. Lett. 2017, 8, 114-125. [CrossRef]

16. Kolinski, A.; Skolnick, J.; Yaris, R. The collapse transition of semiflexible polymers. A Monte Carlo simulation of a model system. J. Chem. Phys. 1986, 85, 3585. [CrossRef]

17. Noguchi, H.; Yoshikawa, K. Morphological variation in a collapsed single homopolymer chain. J. Chem. Phys. 1998, 109, 5070-5077. [CrossRef]

18. Zierenberg, J.; Marenz, M.; Janke, W. Dilute Semiflexible Polymers with Attraction: Collapse, Folding and Aggregation. Polymers 2016, 8, 333. [CrossRef]

19. Seaton, D.T.; Schnabel, S.; Landau, D.P.; Bachmann, M. From Flexible to Stiff: Systematic Analysis of Structural Phases for Single Semiflexible Polymers. Phys. Rev. Lett. 2013, 110, 028103. [CrossRef] 
20. Bhatta, R.S.; Yimer, Y.Y.; Perry, D.S.; Tsige, M. Improved Force Field for Molecular Modeling of Poly(3-hexylthiophene). J. Phys. Chem. B 2013, 117, 10035-10045. [CrossRef]

21. Alessandri, R.; Uusitalo, J.J.; Vries, A.H.d.; Havenith, R.W.A.; Marrink, S.J. Bulk Heterojunction Morphologies with Atomistic Resolution from Coarse-Grain Solvent Evaporation Simulations. J. Am. Chem. Soc. 2017, 139, 3697-3705. [CrossRef]

22. Abraham, M.J.; Murtola, T.; Schulz, R.; Páll, S.; Smith, J.C.; Hess, B.; Lindahl, E. GROMACS: High performance molecular simulations through multi-level parallelism from laptops to supercomputers. SoftwareX 2015, 1-2, 19-25. [CrossRef]

23. Bussi, G.; Donadio, D.; Parrinello, M. Canonical sampling through velocity rescaling. J. Chem. Phys. 2007, 126, 014101. [CrossRef]

24. Jorgensen, W.L.; Maxwell, D.S.; Tirado-Rives, J. Development and Testing of the OPLS All-Atom Force Field on Conformational Energetics and Properties of Organic Liquids. J. Am. Chem. Soc. 1996, 118, 11225-11236.. [CrossRef]

25. Borzdun, N.I.; Larin, S.V.; Falkovich, S.G.; Nazarychev, V.M.; Volgin, I.V.; Yakimansky, A.V.; Lyulin, A.V.; Negi, V.; Bobbert, P.A.; Lyulin, S.V. Molecular dynamics simulation of poly (3-hexylthiophene) helical structure In Vacuo and in amorphous polymer surrounding. J. Polym. Sci. Part B Polym. Phys. 2016, 54, 2448-2456. [CrossRef]

26. Wolf, C.M.; Kanekal, K.H.; Yimer, Y.Y.; Tyagi, M.; Omar-Diallo, S.; Pakhnyuk, V.; Luscombe, C.K.; Pfaendtner, J.; Pozzo, L.D. Assessment of molecular dynamics simulations for amorphous poly(3-hexylthiophene) using neutron and $\mathrm{X}$-ray scattering experiments. Soft Matter 2019, 15, 5067-5083. [CrossRef]

27. Tsourtou, F.D.; Peristeras, L.D.; Apostolov, R.; Mavrantzas, V.G. Molecular Dynamics Simulation of Amorphous Poly(3hexylthiophene). Macromolecules 2020, 53, 7810-7824. [CrossRef]

28. Lee, C.K.; Pao, C.W. Nanomorphology Evolution of P3HT/PCBM Blends during Solution-Processing from Coarse-Grained Molecular Simulations. J. Phys. Chem. C 2014, 118, 11224-11233. [CrossRef]

29. Marrink, S.J.; Tieleman, D.P. Perspective on the Martini model. Chem. Soc. Rev. 2013, 42, 6801-6822. [CrossRef]

30. Campos-Villalobos, G.; Siperstein, F.R.; Patti, A. Transferable coarse-grained MARTINI model for methacrylate-based copolymers. Mol. Syst. Des. Eng. 2019, 4, 186-198. [CrossRef]

31. Flory, P.J. The Configuration of Real Polymer Chains. J. Chem. Phys. 1949, 17, 303-310. [CrossRef]

32. Ivanov, V.; Stukan, M.; Vasilevskaya, V.; Paul, W.; Binder, K. Structures of stiff macromolecules of finite chain length near the coil-globule transition: A Monte Carlo simulation. Macromol. Theory Simulations 2000, 9, 488-499. [CrossRef]

33. Marenz, M.; Janke, W. Knots as a Topological Order Parameter for Semiflexible Polymers. Phys. Rev. Lett. 2016, 116, 128301-128306. [CrossRef]

34. Hu, D.; Yu, J.; Wong, K.; Bagchi, B.; Rossky, P.J.; Barbara, P.F. Collapse of stiff conjugated polymers with chemical defects into ordered, cylindrical conformations. Nature 2000, 405, 1030-1033. [CrossRef]

35. Rodrigues, A.; Castro, M.C.R.; Farinha, A.S.; Oliveira, M.; Tomé, J.P.; Machado, A.V.; Raposo, M.M.M.; Hilliou, L.; Bernardo, G. Thermal stability of P3HT and P3HT: PCBM blends in the molten state. Polym. Test. 2013, 32, 1192-1201. [CrossRef]

36. Bernardi, M.; Giulianini, M.; Grossman, J.C. Self-assembly and its impact on interfacial charge transfer in carbon nanotube/P3HT solar cells. ACS Nano 2010, 4, 6599-6606. [CrossRef]

37. Vukmirovic, N.; Wang, L.W. Electronic structure of disordered conjugated polymers: Polythiophenes. J. Phys. Chem. B 2009, 113, 409-415. [CrossRef]

38. Panzer, F.; Sommer, M.; Baessler, H.; Thelakkat, M.; Koehler, A. Spectroscopic signature of two distinct H-aggregate species in poly (3-hexylthiophene). Macromolecules 2015, 48, 1543-1553. [CrossRef] 